\title{
Modélisations hydrodynamiques sur bathymétries anciennes, une possible alternative aux modélisations morphodynamiques à long terme?
}

\author{
Xavier Bertin $^{(1)}$, Eric Chaumillon ${ }^{(2)}$, Nicolas Pouvreau ${ }^{(3)}$ et Guy \\ Wöppelmann ${ }^{(4)}$
}

${ }^{1}$ Docteur ; ${ }^{2}$ Maître de conférences, ${ }^{3}$ Doctorant, ${ }^{4}$ Maître de conférences. Centre Littoral de Géophysique, Université de La Rochelle, Avenue Michel Crépeau, 17042 La Rochelle cedex1, France ; xavier.bertin@univ-lr.fr

\section{Résumé :}

La dernière décennie a vu le développement intense de systèmes de modélisation morphodynamique dans le but de comprendre et de prévoir l'évolution des systèmes côtiers. Ce type d'approche ne permet pas à l'heure actuelle d'obtenir de résultats satisfaisants lorsqu'il s'agit de simuler des évolutions à long terme (décennie au siècle). Face à ce constat, nous présentons une stratégie alternative, qui consiste à simuler la marée, la houle et le transport sédimentaire en utilisant des données historiques, disponibles pour la Charente Maritime (France) depuis 1824. La comparaison entre les évolutions de l'hydrodynamique et celles de la morphologie apporte notamment des éléments nouveaux dans la compréhension des évolutions séculaires du banc de sable estuarien de la Longe de Boyard.

\section{Abstract :}

An intense development of morphodynamic models has occurred during the last decade, aiming at understanding and predicting coastal system evolutions. Presently, this kind of approach still not allows satisfactory results to be obtained when simulating long-term evolutions (decade to century). Considering this, it is the purpose of this paper to present an alternative strategy, which consists of simulating tides, waves and sediment transport using historical data, available for Charente-Maritime Shorelines (France) since 1824. The comparison between hydrodynamic and bathymetric changes brings new insights in the understandings of long-term evolutions of the Longe de Boyard estuarine sandridge.

\section{Mots clés}

Modélisation hydrodynamique, données historiques, morphodynamique.

\section{Introduction}

Les systèmes de modélisation morphodynamique consistent en un ensemble de modules, permettant la simulation de la marée, de la houle, des transports associés et de 1 'évolution du fond. Ces systèmes ont connu un développement intense au cours de la dernière décennie ${ }^{[9,11,13]}$ et constituent une des perspectives les plus prometteuses pour l'étude des systèmes côtiers. Mis à part quelques récents succès ${ }^{[6,16]}$, ce type d'approche ne permet pas encore de simuler de façon satisfaisante l'évolution des systèmes côtiers pour des échelles de temps longues (décennie au siècle).

Face à ce constat, l'étude que nous présentons ici propose une stratégie alternative, qui consiste à simuler la marée, la houle ou les transports associés pour différents contextes bathymétriques historiques. Les résultats des simulations historiques de la marée ont pu être validées par comparaison avec des enregistrements anciens de marée, eux aussi 
disponibles depuis 1824. La validation des différentes simulations ayant été obtenue avec les mêmes paramètres physiques et numériques, on peut faire l'hypothèse que les différences observées entre chaque simulation ne dépendent que des évolutions de la bathymétrie. Néanmoins, l'ambition de cette méthode n'est pas de reproduire finement l'hydrodynamique et les transports sédimentaires au XIX ${ }^{\text {ème }}$ siècle, mais plutôt d'évaluer dans quelles mesures les évolutions de l'hydrodynamique et des transports d'une simulation à l'autre sont cohérentes avec les évolutions morphologiques à long terme. Dans cette étude, nous montrons comment cette démarche permet d'améliorer la compréhension de l'évolution séculaire et de la dynamique du banc de sable estuarien de la Longe de Boyard ${ }^{[2]}$.

\section{Zone d'étude :}

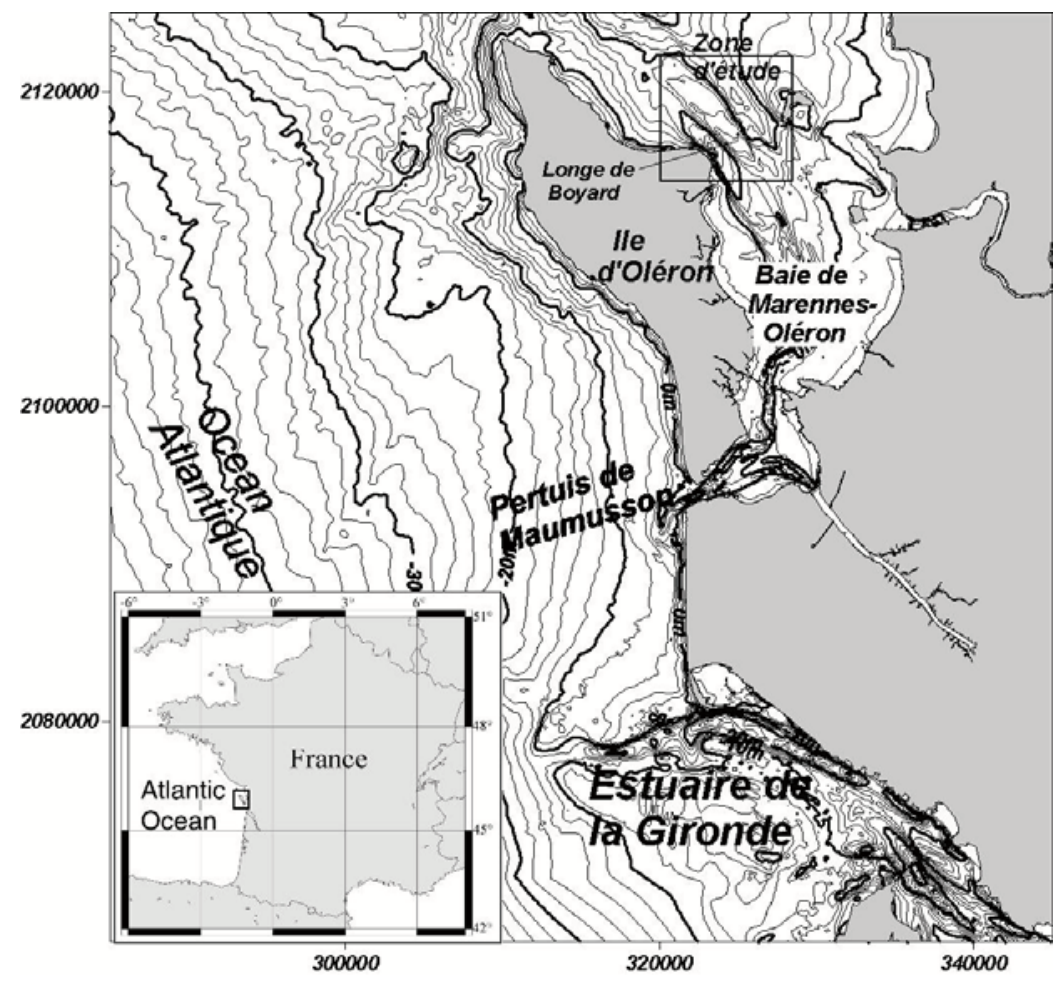

Figure 1. Localisation de la zone d'étude. Les traits gras correspondent aux isobathes $10 \mathrm{~m}$.
La Baie de Marennes-Oléron est une baie tidale de $150 \mathrm{~km}^{2}$, située au milieu de la côte Atlantique Française. Elle possède deux connections avec l'océan Atlantique: l'une au nord, par le pertuis d'Antioche, et l'autre au sud par l'embouchure tidale de Maumusson (Figure 1). L'entrée nord comprend deux chenaux de marée de part et d'autre d'un banc de sable estuarien (La Longe de Boyard) et peut être assimilée à un segment d'estuaire, selon la terminologie de Dalrymple ${ }^{[8]}$.

La marée qui affecte la zone d'étude est de type semi-diurne et varie de moins de $2 \mathrm{~m}$ à plus de $6 \mathrm{~m}$ en vive eaux. Les courants associés atteignent localement $1 \mathrm{~m} / \mathrm{s}$ au niveau de

l'entrée nord et $2 \mathrm{~m} / \mathrm{s}$ au niveau de l'entrée sud.

Les littoraux adjacents à l'embouchure subissent une houle moyenne annuelle de l'ordre $1.5 \mathrm{~m}$, qui peut dépasser $6 \mathrm{~m}$ pendant les fortes tempêtes. La réfraction de la houle autour du nord de l'île d'Oléron lui permet d'atteindre l'entrée nord de la baie de Marennes-Oléron avec une énergie significative ${ }^{[2,3]}$.

\section{Méthodes et données :}

\subsection{Données bathymétriques historiques:}

Plusieurs jeux de données bathymétriques sont disponibles sur toute la zone d'étude depuis 1824 (1824, 1882, 1960-1970 et 2003). Les données les plus anciennes proviennent de la base de données du Service Hydrographique et Océanographique de la Marine (E.P.S.H.O.M.) et ont été digitalisées sur les minutes originales. Les données les plus récentes proviennent des levés de la cellule hydrographique locale (DDE17). Ces données 
ont été géoréférencées à l'aide du S.I.G. Arcview. Le système géodésique NTF a été choisi et des coordonnées projetées Lambert II ont été calculées. La fiabilité de ces données et de cette méthodologie a été vérifiée sur plusieurs plateaux rocheux dans la baie; la précision relative verticale de l'ordre de $35 \mathrm{~cm}$ a déjà permis de quantifier précisément des évolutions morphologiques dans la zone d'étude ${ }^{[2,3,4,7]}$.

\subsection{Enregistrements historiques du niveau de la mer}

Depuis la fin du $18^{\text {ème }}$ siècle, grâce à Charles de Beautemps-Beaupré (1766-1854), père de l'hydrographie moderne, chaque campagne hydrographique s'accompagne d'un levé précis des hauteurs du niveau de la mer. Ces mesures de "marée » visent la réduction des sondes au niveau des plus basses mers astronomiques. Il s'agit d'observations particulières, effectuées à pleine et à basse mer à l'échelle de marée. Nous les avons numérisées, puis nous leur avons appliqué les traitements décrits dans Wöppelmann et al. ${ }^{[15]}$ afin d'en évaluer la qualité. Pour calibrer et valider les modèles hydrodynamiques nous avons également à notre disposition des observations plus classiques de marégraphe, disponibles en 1882 et 1971, respectivement sur la Longe de Boyard et au milieu de la Baie de Marennes-Oléron (figure 1). Il s'agit de hauteurs d'eau horaires pour lesquels de nombreux outils d'analyse existent.

\subsection{Modélisations hydrodynamiques}

\subsubsection{Modélisation de la marée}

Le système telemac2D ${ }^{[10]}$ a été utilisé dans le but de simuler la marée sur la zone d'étude pour quatre configurations bathymétriques différentes (1824, 1882, 1960-70, 2003). Il s'agit d'un modèle aux éléments finis, qui résout les équations de Navier-Stokes, moyennées sur la hauteur d'eau. La discrétisation en espace a été réalisée grâce à un maillage triangulaire où la taille des mailles varie de $3000 \mathrm{~m}$ au large à $50 \mathrm{~m}$ au niveau des chenaux de marée de la zone d'étude. Le domaine modélisé s'étend de l'estuaire de la Gironde vers le sud, au nord de l'île de Ré vers le nord tandis que la limite au large décrit un arc de cercle de $60 \mathrm{~km}$ autour de l'île d'Oléron. Les forçages du modèle au niveau des conditions aux limites incluent: (1) La marée, calculée à chaque pas de temps à partir d'un série de 30 points disposés le long de la frontière ouverte et pour lesquels les amplitudes et phases des 17 harmoniques principales de la marée ont été pris en compte;(2) les débits des fleuves Charente et Gironde, pour lesquels les valeurs annuelles moyennes ont été considérées.
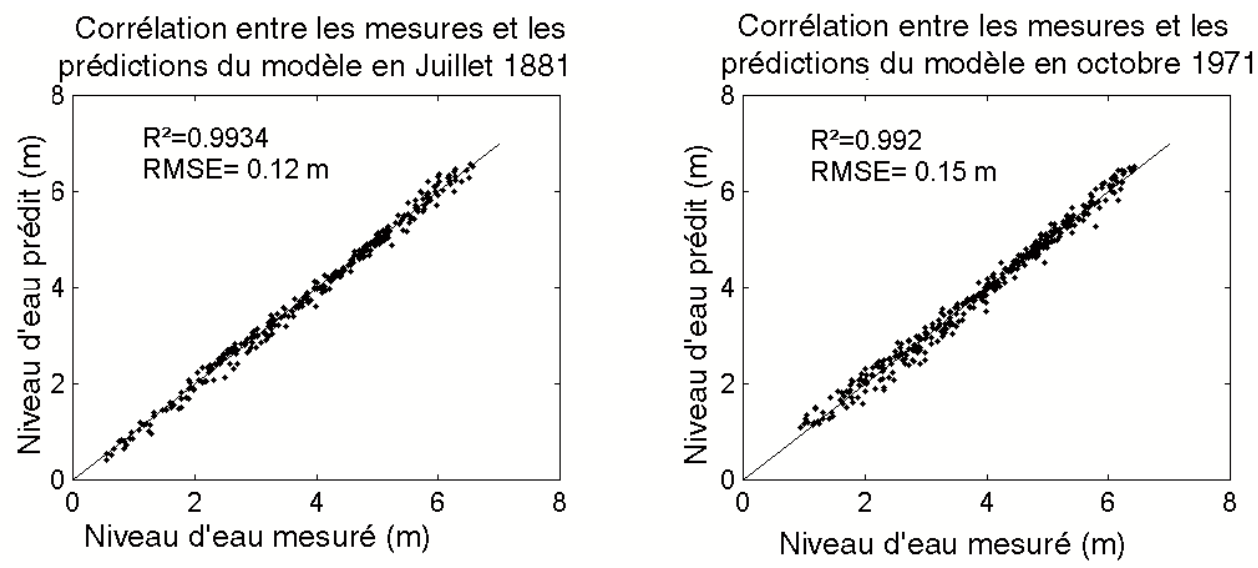

Figure 2. Corrélation entre les hauteurs prédites par le modèle et mesurées en 1881 sur la Longe de Boyard et en 1971 au niveau du pont d'Oléron, montrant une corrélation excellente pour ces deux dates. 
La calibration du modèle sur la bathymétrie contemporaine a été effectuée par comparaison avec des données de hauteur d'eau et des données ADCP disponibles aux deux entrées de la baie de Marennes-Oléron. Les simulations de 1960-70, 1882 et 1824 ont été lancées avec les mêmes conditions aux limites, les mêmes conditions initiales et les mêmes paramètres physiques et numériques. La validation de ces nouveaux résultats a été obtenue par comparaison avec des enregistrements de marée historiques disponibles en 1824,1882 et 1971 (figure 2).

\subsubsection{Modélisation du transport associé à la marée en 1824}

Les résultats de hauteur d'eau et de vitesse des courants calculés par Telemac2D pour la bathymétrie de 1824 ont ensuite été couplés aux équations de transport par charriage proposées par Van Rijn ${ }^{[14]}$ en vue de calculer le transport résiduel potentiel du sable sur un cycle vive eau/morte eau. Ce calcul prend en compte une granulométrie qui varie dans l'espace, déduite de mesures historiques au plomb suiffé, datant de 1824.

\subsubsection{Modélisation de la propagation de la houle}

Le modèle de houle spectral SWAN a été choisi dans cette étude et résout l'équation de conservation spectro-angulaire d'énergie de la houle en domaine côtier ${ }^{[5]}$. Ce modèle a été paramétré pour rendre compte : (1) de la réfraction de la houle ; (2) de la diffraction de la houle; (3) du déferlement bathymétrique; (4) de la dissipation d'énergie par frottement sur le fond; (5) de l'interaction avec les courants de marée, calculés à l'aide des simulations historiques Telemac2D; (6) des interactions entre triplets de fréquence. La validation du modèle a été obtenue par ajustement du coefficient de frottement et par comparaison avec des mesures effectuées dans le Pertuis d'Antioche en $2002^{[12]}$.

\section{Evolutions morphologiques et hydrodynamiques de la zone d'étude :}

\subsection{Evolutions morphologiques séculaires}

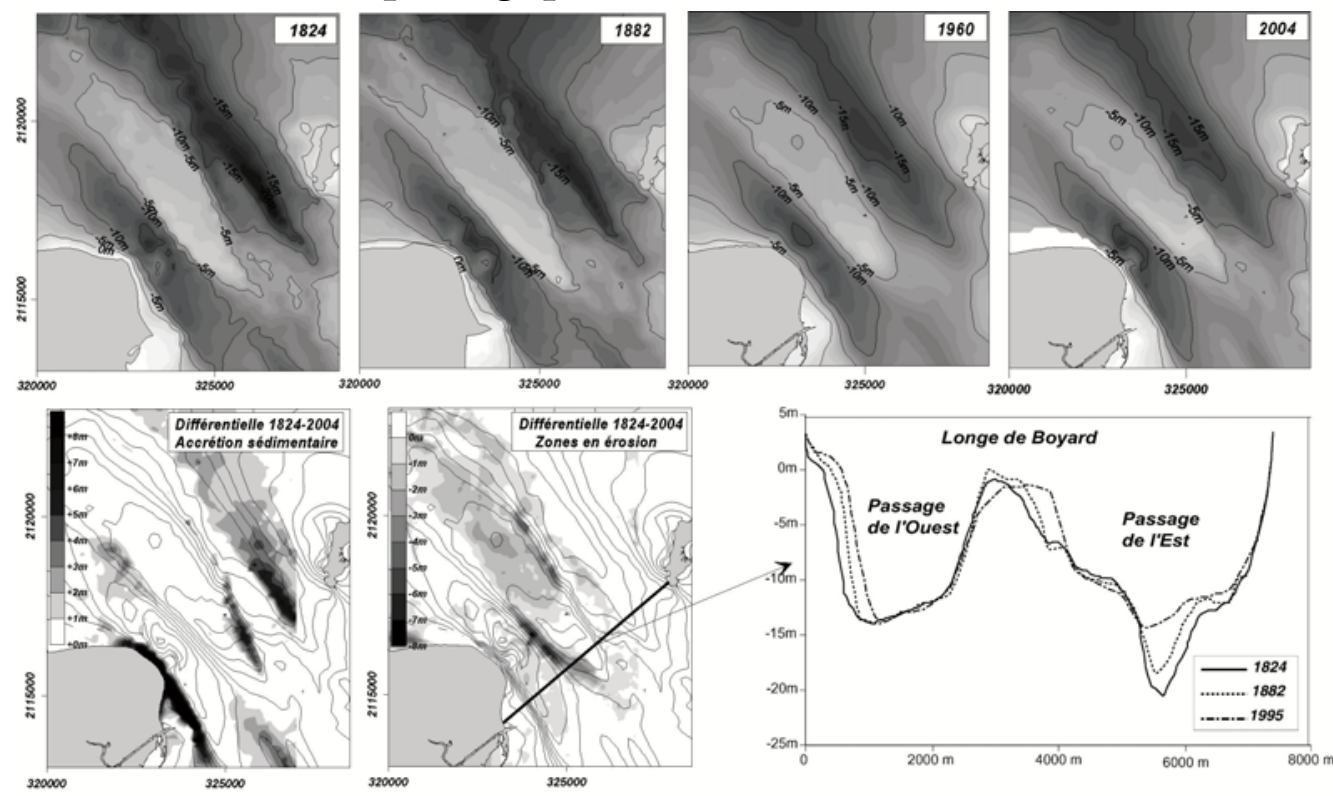

Figure 3 . Modèles numériques de terrain de l'entrée nord de la baie de Marennes-Oléron en 1824, 1882, 1960 et 2004 et cartes de différence de bathymétrie montrant les évolutions de la morphologie entre 1824 et 2004.

Quatre jeux de données bathymétriques sont disponibles au niveau de l'entrée nord de la baie de Marennes-Oléron et ont permis d'étudier ses évolutions morphologiques depuis 1824, dont les plus importantes sont (figure 3):

- Un fort comblement par aggradation du chenal de marée oriental 
- Une migration vers le NE du chenal occidental associée à la progradation de la côte $\mathrm{N}$ de l'île d'Oléron.

- La présence de zones en érosion et en accrétion au niveau des flancs NE et SW du banc de sable, entraînant son évolution depuis une forme elliptique vers une forme sigmoïde.

- L'érosion du toit du banc dans sa partie NW.

- Le bilan sédimentaire du banc entre 1824 et 2001 est négatif.

4.2. Transport résiduel du sable en 1824 et comparaison avec les évolutions morphologiques séculaires

Transport résiduel en 1824

laccrétion sédimentaire entre 1824 et 2004

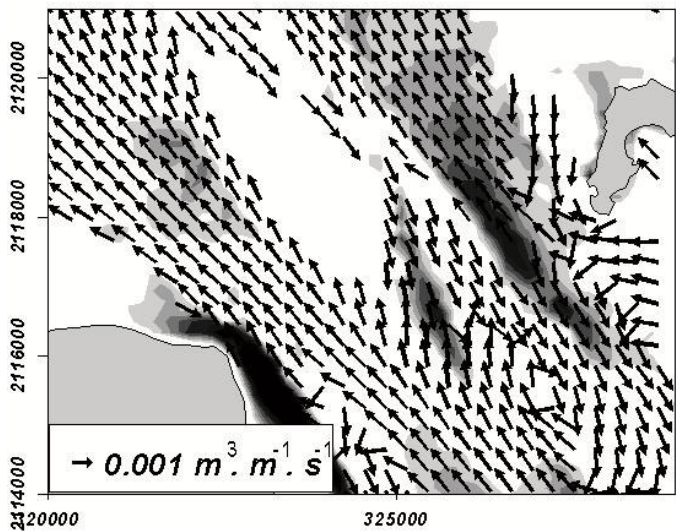

Transport résiduel en 1824

/érosion entre 1824 et 2004

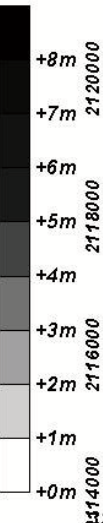

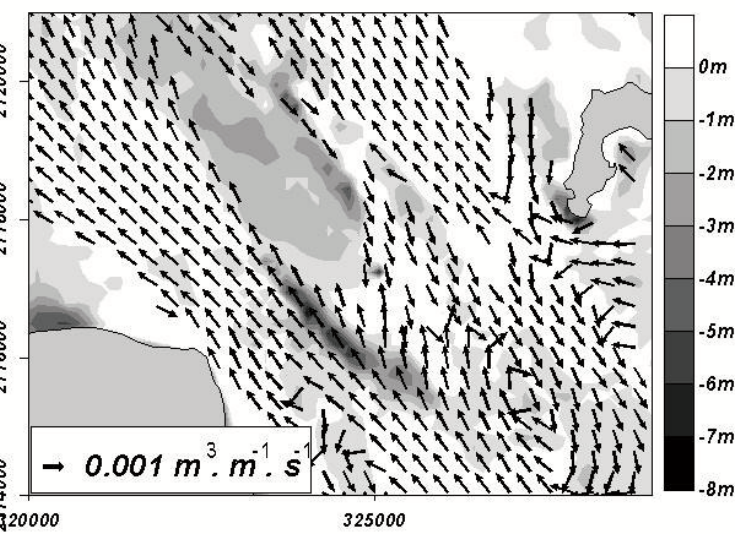

Figure 4.. Transport résiduel en 1824 sur la Longe de Boyard, superposé à la différence de bathymétrie entre 1824 et 2004. Résultat issu de la simulation Telemac2D sur la bathymétrie de 1824, couplée avec les équations de transport de Van Rijn (1984).

Au niveau de la Longe de Boyard, la modélisation du transport résiduel de sable, sur la bathymétrie de 1824, montre que ce banc a son flanc NE soumis à un transport résiduel orienté dans le sens du flot et son flanc SW soumis à un transport résiduel orienté dans le sens du jusant (figure 4). La zone majeure d'accrétion sédimentaire entre 1824 et 2004, à l'extrémité SE du banc, se superpose à un lieu de convergence du transport résiduel par la marée pour chaque simulation historique, les vecteurs de transport effectuant une déviation horaire vers la crête du banc. La moitié NW du toit du banc subit une érosion mais n'est pas un lieu de transport résiduel sous l'effet des courants de marée.

\subsection{Propagation de la houle en $\mathbf{1 8 2 4}$}
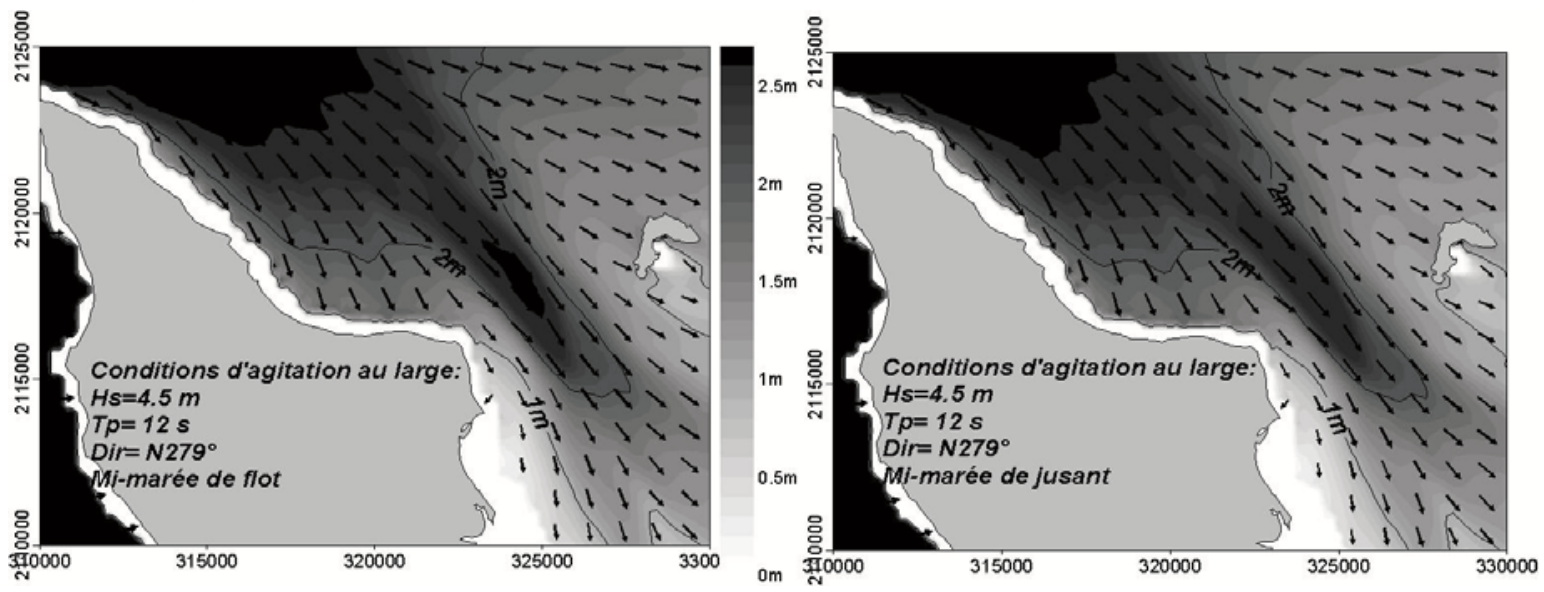

Figure 5 . Simulation de la propagation de la houle sur la bathymétrie de 1882 pour des conditions de houle au large énergétique $\left(\mathrm{Hs}=4.5 \mathrm{~m}, \mathrm{Tp}=12 \mathrm{~s}\right.$ et Dirp $\left.=\mathrm{N} 275^{\circ}\right)$, à mi-marée de flot et de jusant. Les vecteurs représentent la direction de propagation de la houle. 
La simulation numérique de la propagation de la houle à l'aide du modèle SWAN sur la bathymétrie de 1824 montre tout d'abord la réfraction de la houle autour de la pointe nord de l'île d'Oléron, qui lui permet d'atteindre des zones géographiquement abritées (figure 5), comme l'entrée nord de la Baie de Marennes-Oléron. Au niveau de cette dernière, la forme allongée de la Longe de Boyard génère une forte réfraction convexe de la houle, ce qui amplifie fortement son amplitude. La simulation de la propagation de la houle au moment du flot montre que les courants de flot accentuent légèrement ce phénomène au niveau du toit du banc.

\section{Discussion}

\subsection{Evolution des chenaux en réponse à l'évolution des débits tidaux}

L'analyse des sections mouillées au niveau de l'entrée nord de la baie de MarennesOléron a montré une diminution de $15 \%$ entre 1824 et 2004, principalement liée au fort comblement du chenal de marée oriental. Le comblement de ce chenal pourrait s'expliquer par un réajustement de sa section, en réponse à une diminution des prismes tidaux de 5 à $10 \%$ depuis 1824 , mise en évidence par les simulations historiques de la marée. Cette diminution des prismes de marée aux deux entrée de la baie de MarennesOléron a été expliquée par le comblement sédimentaire de la baie de Marennes-Oléron depuis $1824^{[3]}$.

\subsection{Evolution du banc sous l'action des courants de marée}

La simulation du transport résiduel du sable sur les bathymétries de 1824 et 1882 montre que le flanc NE du banc était soumis à un transport dirigé dans le sens du flot alors que le flanc SW était soumis à un transport dans le sens du jusant (figure 4). L'accrétion sédimentaire mise en évidence au niveau de l'extrémité Sud-est du banc coïncide avec une zone de convergence du transport résiduel, liée à la déviation des vecteurs de transport vers la crête du banc (figure 4). Cette convergence des vecteurs de transports n'est pas liée à une différence de friction, liée à une différence de hauteur d'eau entre les chenaux et le banc, car pour toutes les simulations, le changement de direction des vecteurs de transport ne se produit que dans le sens horaire (figure 4). L'hypothèse d'un contrôle par la force de Coriolis a également été écartée en supprimant les termes de Coriolis dans Telemac2D et en obtenant une forte déviation horaire des courants de marée (figure 6).

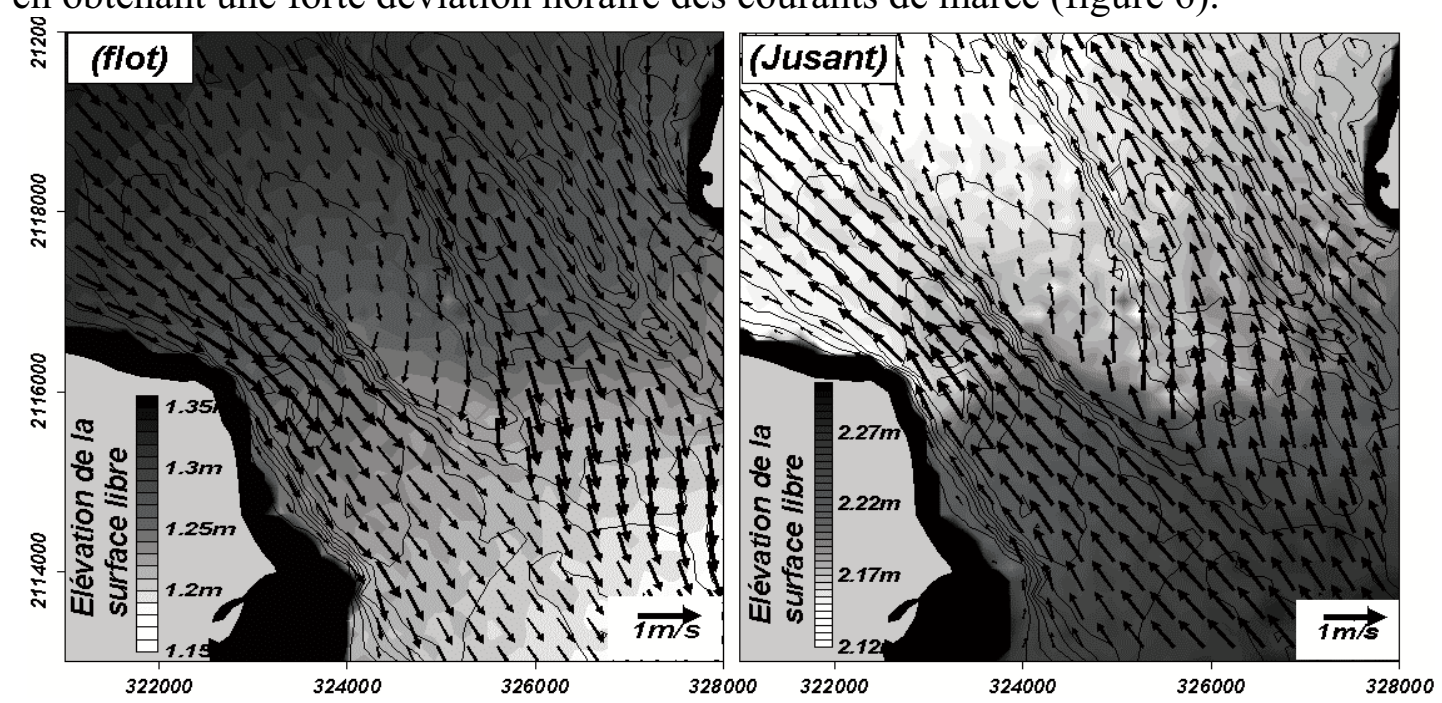

Figure 5- Isovaleurs de la surface libre, lors du flot et du jusant au niveau de la Longe de Boyard en 1882, expliquant la déviation systématiquement horaire des courants de marée. Résultat issu de la simulation numérique Telemac2D sur la bathymétrie de 1882, modifié d'après Bertin et Chaumillon (sous presse-b). 
L'analyse de la hauteur de la surface libre montre par contre un gradient de part et d'autre de la crête SE du banc pour de faibles hauteur d'eau (figure 6), qui peut s'expliquer par la légère obliquité de cette partie du banc par rapport aux courants dans les chenaux. Ce gradient pourrait expliquer la déviation des courants vers la crête du banc, qui conduit à une convergence du transport résiduel et expliquerait ainsi l'accrétion sédimentaire entre 1824 et 2003.

\subsection{Evolution de l'entrée nord sous l'action de la houle}

L'érosion sur la moitié NW du toit n'est associée à un transport résiduel par les courants de marée pour aucune des simulations (1824, 1882, 1960, 2003), la compétence des courants de marée étant systématiquement trop faible pour générer un transport significatif des sables. La houle, qui pénètre la partie interne du Pertuis d'Antioche et déferle sur cette partie du banc, pourrait par contre expliquer l'érosion de ce secteur. Les résultats des simulations historiques de propagation de la houle dans la baie de MarennesOléron (figure 5) confirment cette hypothèse, en montrant par exemple que pour une houle au large de 4,5 $\mathrm{m}$ (sur l'année, 5 à $7 \%$ des houles au large sont supérieures à cette valeur), des vagues de près de $3 \mathrm{~m}$ atteignent la Longe de Boyard en 1824 comme pour la bathymétrie contemporaine. La forme allongée du banc de sable par rapport aux crêtes de houle entraîne en effet une forte réfraction convexe, qui augmente de façon significative l'amplitude de la houle au niveau du toit du banc par rapport à celle dans les chenaux. Ce phénomène est légèrement amplifié au moment du flot et pourrait entraîner un important transport de sédiments depuis le toit du banc vers le SE de la Longe de Boyard et la baie de Marennes-Oléron.

\section{Conclusion}

La modélisation sur bathymétries historiques de la marée, du transport associé et de la houle permet d'expliquer la plupart des évolutions morphologiques séculaires de l'entrée nord de la baie de Marennes-Oléron depuis 1824. Elle permet en outre de proposer le mécanisme de convergence du transport du sable, sous l'effet d'un gradient de la surface libre, qui constitue un processus nouveau dans la compréhension des bancs de sable estuariens. Toutefois, cette approche de modélisation, simpliste par rapport aux systèmes morphodynamiques souvent très complexes, ne nous permet pas d'envisager de façon fiable les évolutions futures de l'entrée nord de la baie de Marennes-Oléron. La méthodologie présentée dans cette étude constitue donc un outil très simple, qui permet de mieux comprendre les évolutions séculaires de tels systèmes côtiers complexes, et qui présente encore de nombreuses perspectives, en attendant que les systèmes de modélisation morphodynamiques ne soient au point sur les échelles de temps séculaires.

\section{Remerciements :}

Cette recherche est financée en partie par le conseil général de la Charente-Maritime (bourse doctorale de N. Pouvreau) et par le Groupe de Recherches en Géodésie Spatiale. Les bathymétries et les composantes harmoniques de la marée proviennent de l'EPSHOM, les observations du niveau de la mer datant de 1882 et 1973 sont également fournies par l'EPSHOM dans le cadre du système SONEL (http://www.sonel.org).Les auteurs tiennent à remercier à M. Fardet, conservateur du Service Historique de la Défense - Département de la Marine, Antenne de Rochefort ainsi qu'à tout le personnel pour l'aide apportée dans la recherche des cahiers des missions hydrographiques. 


\section{Références}

1 Beautemps-Beaupré C. et P. Daussy, 1829. Exposé des travaux relatifs à la reconnaissance hydrographique des côtes occidentales de France. Imprimerie Royale, Paris.

2 Bertin X. And Chaumillon E., 2005. Apports de la modélisation sur bathymétries historiques dans la compréhension des évolutions des bancs de sable estuariens, C.R. geosciences 337, 13751383.

3 Bertin X., Chaumillon E., Sottolichio, A. And Pedreros, R., 2005. Tidal inlet response to sediment infilling of the associated bay and possible implications of human activities: The Marennes-Oléron Bay and Maumusson Inlet, France, Continental Shelf Research 25, 1115-1131.

4 Bertin X, Chaumillon E, Weber N And Tesson M, 2004. Morphological evolution and time-varying bedrock control of main channel at a mixed energy tidal inlet: the Maumusson inlet, France, Marine Geology 204, 187-202

5 Booij, N., Ris, R.C. and Holthuijsen, L.H., 1999. A third generation wave model for coastal regions; Part I: model description and validation. Journal of Geophysical Research 104, 7649-7666.

6 Cayocca, F., 2001. Long-term morphological modeling of a tidal inlet the Arcachon basin, France. Coast. Eng. 42, 115-142.

7 Chaumillon, E., Gillet, H., Weber, N., Walker, P. And Tesson, M., 2002. Evolution temporelle et architecture interne d'un banc sableux estuarien : la Longe de Boyard (littoral Atlantique, France). C.R. Geosciences, 334, 119-126.

8 Dalrymple, R.W., Zaitlin, B.A. and Boyd, R., 1992. Estuarine facies models : conceptual basis and stratigraphic implications. Jour. of Sed. Petrol., 62, 1030-1043.

9 Davies, A.G., L.C. van Rijn, J.S. Damgaard, J. Van de Graaff and J.S. Ribberink (2002). Intercomparison of research and practical sand transport models, Coast. Eng., 46/1: 1-23.

10 Hervouet, J.M., Van Haren, L., 1994. Telemac 2-D Version 3.0 Principle Note, EDF-DER report HE43/94/052/B.

11 Holliday, B.W., E.C. McNair and N. C. Kraus (2002). The U.S. Army Corps of Engineers' Coastal Inlets Research Program, Proceedings Dredging '02, ASCE.

12 Idier, D., Pedreros, R., Oliveros, C., Sottolichio, A., Choppin, L., et Bertin, X., 2006. Importance de la houle dans la dynamique hydro-sédimentaire des Pertuis Charentais, sous presse au C.R. Géosciences.

13 Nicholson, J.I., Broker, J.A., Price, D., Tanguy, L., 1997. Intercomparison of coastal area morphodynamic models, Coast. Eng. 31 (1), 97-123.

14 Van Rijn, L.C., 1984. Sediment transport. Part I: bedload transport. Proc. ASCE Journ. Of Hyd. Div. 110 (HY10), 1431

15 Wöppelmann G., N. Pouvreau et B. Simon, 2006. Brest sea-level record : a time series construction back to the 18th century. Ocean Dynamics, DOI 10.1007/s10236-005-0044-z.

16 Work, P.A, Guan, J., Hayter, E.J. et Elçi, S., 2001. Mesoscale model for morphologic change at tidal inlets. Journ. of Wat., Port, Coast. Ocean Eng. 127 (5), 282-289. 